\title{
Using Observed Contrast Motion During Cardiac Catheterization to Tune Patient Specific Coronary Blood Flow Simulations
}

\author{
S Moore ${ }^{1}$, K Halupka ${ }^{1}$, S Zhuk $^{2}$ \\ ${ }^{1}$ IBM Research, Melbourne, Australia \\ ${ }^{2}$ IBM Research, Dublin, Ireland
}

\begin{abstract}
In the assessment of coronary artery disease, virtual Fractional Flow Reserve is an emerging technology that uses patient specific of Computational Fluid Dynamics simulations to infer the pressure loss through a stenosis, replacing an effective, but expensive technique based on the use of a pressure wire. To date, most vFFR efforts make use of reduced order lumped parameter models for the inlet and outlet/s of the coronary arterial tree to approximate the patient specific boundary conditions, but suffer from the inability to specify the associated parameters in a patient specific manner. When applying $v F F R$ in a catheter laboratory setting using X-Ray angiograms as the basis for creating the geometrical model, there is some indirect functional information available through observing the motion of the radio-opaque contrast agent. In this work, we present a novel method for tuning the arterial resistances, based on simulating the contrast release and minimising a misfit defined in terms of simulated and observed arrival times of the contrast at multiple points within a coronary tree.
\end{abstract}

\section{Introduction}

Virtual (vFFR) is an emerging technology that uses computational fluid dynamics to simulate the hemodynamics in a personalised digital model of a coronary tree and provide the pressure drop through a coronary stenosis. Current efforts in this space use either Computed Tomography Coronary Angiography (CTCA) or Invasive Coronary Angiography (ICA) to build these digital models. In order to create a faithful representation of the hemodynamics, accurate boundary conditions are important and many efforts in this space include 0D lumped parameter models representing the remainder of the cardiovascular system and coronary tree. Use of these models requires specification of the individual arterial resistances and capacitances, which are generally not known on a patient specific basis however. In the context of ICA, there is some additional functional information in the observed motion of the contrast front through the coronary tree, that can be used to indirectly infer these parameters. In this work we present a proof of principle method that simulates the fluoroscopic process and provides a control system to match the observed and simulated contrast motion.

\section{Methods}

As the process of geometric reconstruction of personalised model from pairs of angiograms that would be acquired during ICA is outside the scope of the present work, the computational models of the left and right coronary trees are based on an existing centreline model segmented from a CTCA dataset [1]. The centrelines (Figure 1) were passed to a 'polyballModelling' filter [2] to generate a 3D image defining voxels within the arterial lumen, then a contouring filter to generate a triangulated surface representing the arterial wall, that was subsequently clipped and had inlet and outlet end caps generated using end points from the original centreline and filters from the Visualisation Toolkit [3]. The hemodynamics is modelled by solving the incompressible Navier-Stokes equations:

$$
\begin{aligned}
\nabla \cdot \mathbf{u} & =0 \\
\frac{\partial \mathbf{u}}{\partial t}+\nabla \cdot(\mathbf{u u}) & =\nabla \cdot(\nu \nabla \mathbf{u})-\nabla \mathbf{p}
\end{aligned}
$$

where $\mathbf{u}(\mathbf{x}, \mathbf{t}), p(\mathbf{x}, \mathbf{t})$, and $\nu$ are the velocity and kinematic pressure and viscosity respectively. In order to capture some of the key phenomena exhibited by coronary hemodynamics, two lumped parameter (LP) models are used for the specification of inlet and outlet pressures respectively [4]. At the inlet, the cardiovascular model of Simaan et al. [5]. At each outlet, the coronary outlet model of Sankaran et al. [6] was used:

$$
\begin{aligned}
C_{a} \frac{d p_{o}}{d t} & =\frac{\left(p_{d}-p_{o}\right)}{R_{p}}+Q_{a} \\
C_{i m} \frac{d p_{d}}{d t} & =\frac{\left(p_{o}-p_{d}\right)}{R_{p}}-\frac{p_{d}}{R_{d}}+C_{i m} \dot{p}_{l v}
\end{aligned}
$$




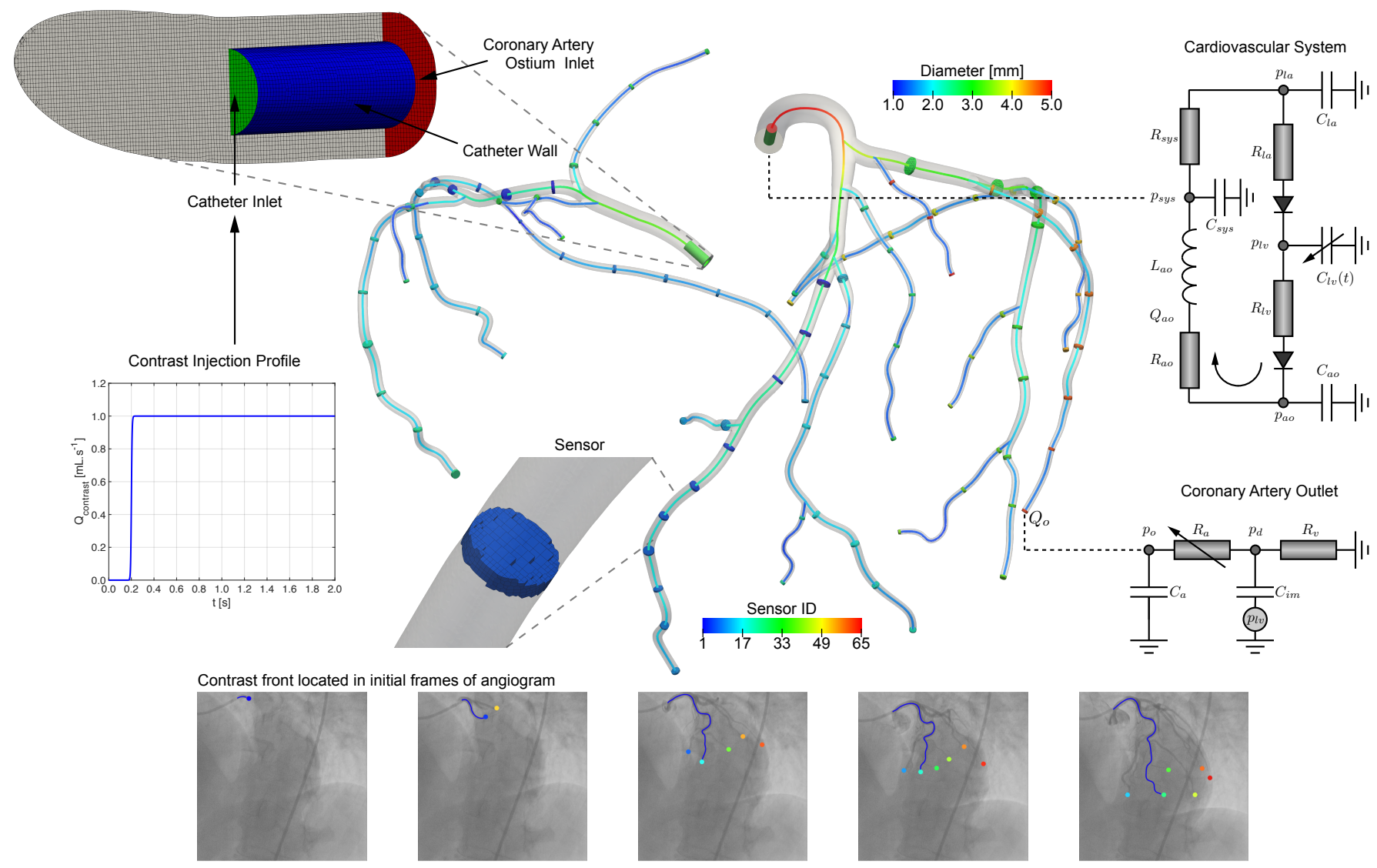

Figure 1. The coronary arterial models used in the present work illustrating the centrelines, catheter inlet, contrast release profile, lumped parameter models, and an example of contrast front location in a subset of angiogram frames to define a set of sensors.

where $p, Q, R, C$ denote the pressure, flowrate, resistance, and capacitance, and the subscripts $o, l v, i m, a$, and $v$ denote the outlet, left atrium and ventricle, systemic, aortic, intramyocardial, coronary arterial, and venous compartments respectively (Figure 1). Parameters are taken from [4] and each LP model is explicitly coupled as a Dirichlet pressure boundary condition, solved using the Runge-Kutta method with a time step size of $1.0 \times 10^{-6} \mathrm{~s}$. ICA is performed by injecting a specified volume of radiocontrast agent at the root of the coronary tree, but not completely obstructing normal coronary blood flow. This contrast agent is modelled by solving a scalar transport equation:

$$
\frac{\partial c}{\partial t}+\nabla \cdot(\mathbf{u c})=\nabla \cdot(\kappa \nabla \mathbf{c})
$$

in addition to the Navier-Stokes equations [7, 8], where $c(\mathbf{x}, \mathbf{t})$ is the concentration $\left[\mathrm{mol} . \mathrm{m}^{-3}\right]$ with mass diffusivity $\kappa=1.0 \times 10^{-6} \mathrm{~m}^{2} \cdot \mathrm{s}^{-1}$ [8]. Contrast injection is simulated by controlled contrast flowrate, defining a Dirichlet condition for $\mathbf{u}$ and $c$ on the catheter inlet. The control approach is based on the idea that the contrast front can be located in the initial frames of a standard coronary angiogram and located as a 'sensor' point on a reconstructed 3D model with a corresponding 'arrival time', relative to the point where the injection of contrast began (Figure 1). As such, the coronary trees are equipped with artificial sensors that record the concentration averaged over their volume, which is used to define their activation. To match the observed and simulated arrival times, we aim to find resistance values $R_{a, o}$ for each outlet $o$, such that the observed arrival time $t_{s}^{\text {obs }}$ and the simulated arrival time at $t_{s}^{\mathrm{sim}}$ at sensor $s$, are as close as possible for all sensors. The control approach is motivated by the observation that over a relatively wide range of arterial resistances, despite the complex pulsatile flow and mixing of blood and contrast, occurring, the arrival time of the contrast agent at individual sensors follows an approximately linear relationship to arterial resistance (Figure 2(a)-2(b)). We therefore assume that for $s \in \mathcal{S}, t_{s}^{\text {sim }} \approx \beta_{0, s}+\sum_{o=1}^{N} \beta_{o, s} R_{a, o}$ for some $\beta$ values. This modelled linear approximation we refer to as $t_{s}^{\text {mod }}$. The optimal set of $R_{a, o}$ values will be those which cause the simulated flowfield to be closest to the observed flowfield. For the loss function we consider the $L_{\infty}$ norm 


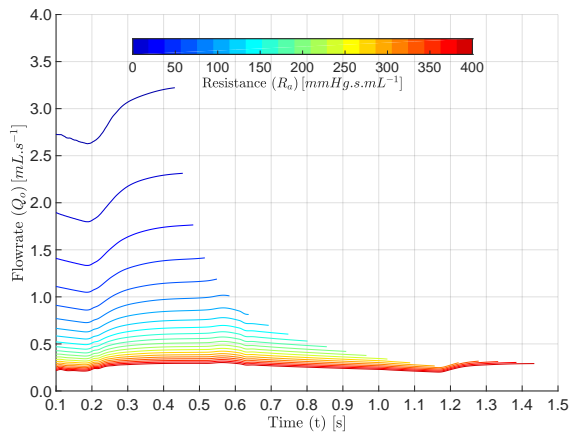

(a)

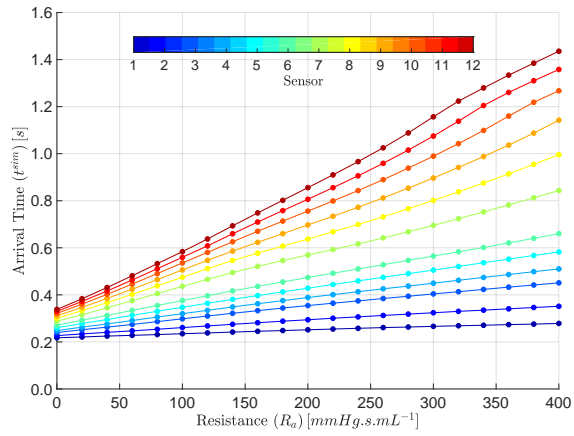

(b)

Figure 2. A set of simulations where the arterial resistance is varied uniformly for each outlet. (a) illustrates the variation in flowrate at one example outlet (b) illustrates the variation in arrival time of the contrast agent at the set of sensors along the path from the inlet to one example outlet as a function of arterial resistance.

of the relative difference between $t_{s}^{\text {sim }}$ and $t_{s}^{\text {obs }}$ :

$$
L_{\infty}\left(R_{a}\right)=\max _{s \in \mathcal{S}} \frac{\left|t_{s}^{\mathrm{sim}}-t_{s}^{\mathrm{obs}}\right|}{t_{s}^{\mathrm{obs}}}
$$

Using $t_{s}^{\text {mod }}$ as a proxy for $t_{s}^{\text {sim }}$ in the loss function, we obtain an optimisation problem (linear program) with variables representing the choices of $R_{a, o}$ as well as the resulting predicted times $t_{s}^{\text {mod }}$ and absolute errors $e_{s}$ for each sensor $s$. An additional variable $\eta$ represents the $L_{\infty}$ norm.

$$
\begin{array}{ll}
\min _{t_{s}^{\text {mod }}, R_{a}, e} \sum_{s \in \mathcal{S}} e_{s}+\eta & \\
\text { s.t. } e_{s} \geq t_{s}^{\text {mod }}-t_{s}^{\text {obs }} & \forall s \in \mathcal{S} \\
e_{s} \geq t_{s}^{\text {obs }}-t_{s}^{\text {mod }} & \forall s \in \mathcal{S} \\
\eta \geq e_{s} & \forall s \in \mathcal{S} \\
t_{s}^{\text {mod }}=\beta_{0, s}+\sum_{o=1}^{N} \beta_{o, s} R_{a, o} & \forall s \in \mathcal{S} \\
0 \leq R_{a, o} \leq R_{a, \text { max }} & \forall o \in 1 \ldots N
\end{array}
$$

The process of tuning a simulation begins by initialising the arterial resistances with random values, constrained to lie within a range that will only allow for physiologically realistic flows (11). Multiple 'forward simulations' of the contrast motion are performed, recording the arrival times at the sensors and updating resistances. When there is less data than required to fit the full model, we do a limited linear regression where we model the arrival time at sensor $s$ as depending only on the $R_{a, o}$ values 'downstream' of $s, \mathcal{D}_{f}, t_{s}^{\text {mod }}=\beta_{0, s}+\sum_{o \in \mathcal{D}} \beta_{o, s} R_{a, o}$. When we have enough information to define the relationship between arrival times and resistances as well formed linear regression problems to determine $\beta$, we can then iterate between solv-

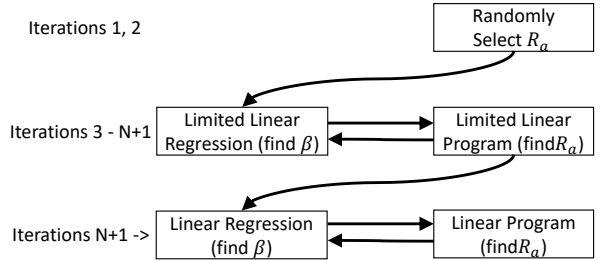

Figure 3. Schematic view of the optimisation process, using linear programming to find $R_{a}$ values and linear regression to find $\beta$ values. Initially, $R_{a}$ values are randomly selected.

ing the optimisation problem to find trial $R_{a}$ values and using linear regression to find $\beta$ values.

\section{Results}

To demostrate the contrast based tuning process, Figures 4(a) and 4(b) present results for five different tuning runs on the Left coronary tree, possessing 13 terminal segments, illustrating the variation in misfit and the converged set of arterial resistances for random initialisations. The average relative error in the converged resistances is $0.7 \%$ with a maximum error of $4.2 \%$ occurring at outlet 6 in the second optimsation run. Figures 4(c) and 4(d) present results for five different tuning runs on the Right coronary tree, possessing 7 terminal segments. The average relative error in the converged resistances is $0.2 \%$ with a maximum error of $0.8 \%$ occurring at outlet 7 in the first optimsation run.

\section{Conclusion}

This work demonstrates a proof of principle that observing and simulating the physics of contrast motion could provide a means to infer the coronary arterial resistance distribution in order to improve the patient specificity of vFFR simulations. Future work will seek to implement 


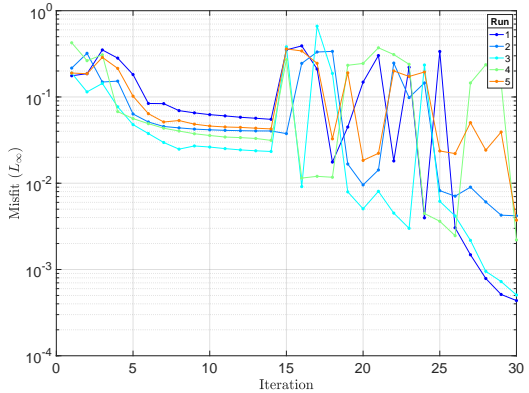

(a)

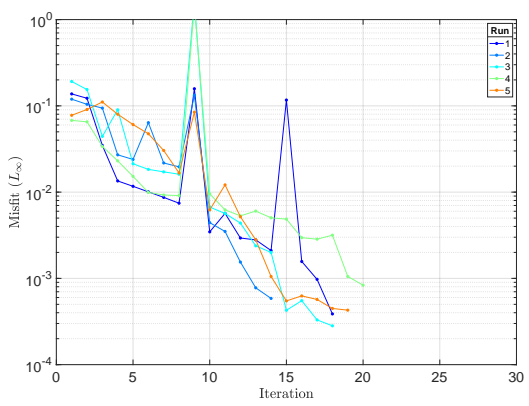

(c)

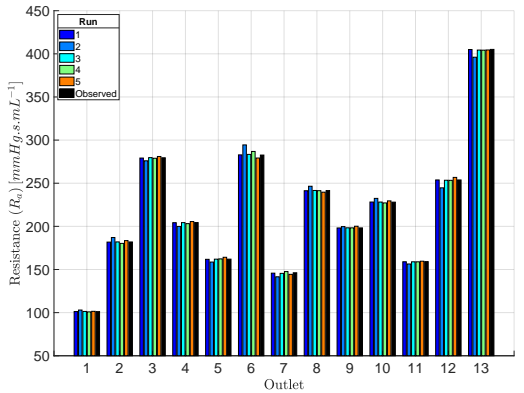

(b)

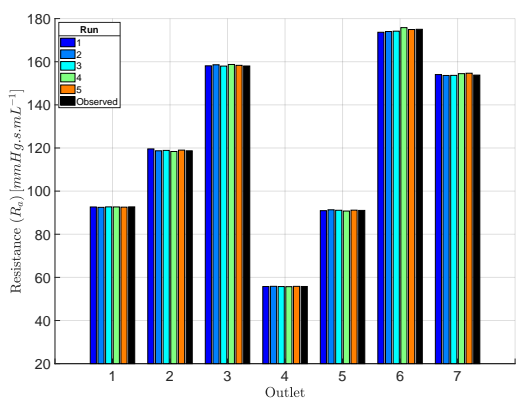

(d)

Figure 4. Convergence results from a contrast-based tuning simulation, (a) illustrates the variation in misfit and (b) illustrates the variation in the set of arterial during the forward simulations for the left coronary treen and (c) and (d) illustrate the same results for the right coronary tree.

analytics for performing the contrast tracking in real angiographic images and performing experimental validation in synthetic phantom models.

\section{References}

[1] Corporation OSMS. http://www.vascularmodel.com.

[2] Antiga L, Piccinelli M, Botti L, Ene-Iordache B, Remuzzi A, Steinman DA. An image-based modeling framework for patient-specific computational hemodynamics. Med Biol Eng Comput Nov 2008;46(11):1097-112.

[3] Schroeder W, Lorensen B, Martin Ken, Kitware I. The visualization toolkit : an object-oriented approach to 3D graphics. 4th ed edition. [Clifton Park, N.Y.] : Kitware, 2006. ISBN 9781930934191. "Copyright 1993-2006 : Ken Martin, Will Schroeder, Bill Lorensen”-Disc label.

[4] Alena Jonasova JV, Bublik O. Blood flow simulations in patient-specific aorto-coronary bypass models: The role of boundary conditions. VI International Conference on Computational Bioengineering, 2015; .

[5] Simaan MA. Rotary Heart Assist Devices. Berlin, Heidelberg: Springer Berlin Heidelberg. ISBN 978-3-540-78831-7, 2009; 1409-1422.

[6] Patient-specific multiscale modeling of blood flow for coronary artery bypass graft surgery. Annals of Biomedical Engineering Oct 2012;40(10):2228-2242. ISSN 1573-9686.

[7] Ford MD, Stuhne GR, Nikolov HN, Habets DF, Lownie
SP, Holdsworth DW, Steinman DA. Virtual angiography for visualization and validation of computational models of aneurysm hemodynamics. IEEE Trans Med Imaging Dec 2005;24(12):1586-92.

[8] Durant J, Waechter I, Hermans R, Weese J, Aach T. Toward quantitative virtual angiography: Evaluation with in vitro studies. In 2008 5th IEEE International Symposium on Biomedical Imaging: From Nano to Macro. ISSN 19457928, May 2008; 632-635.

Address for correspondence:

\section{Stephen Moore}

IBM Research Australia

Level 22, 60 City Rd, Southbank, VIC 3006, Australia tel. +61450009534

stevemoore@au1.ibm.com 